\title{
Numerical Analysis of Coating Device based on Fluent
}

\author{
Chen Zhe, Bai Ziyan, Chen Xinxuan \\ School of Construction Machinery, Chang'an University, Xi'an 71006, Shaanxi, China
}

Keywords: Extrusion coating; Extrusion die; Inner flow channel; Uniformity.

Abstract: According to the applicable conditions of coating quality of two different coating models in the extrusion coating of lithium battery, the flow state of the slurry in the inner flow channel of the extrusion die is simulated by Fluent software. The simulation results show that the coating uniformity of the two models basically meets the requirements. The FLUID1 die is superior to the FLUID2 die in terms of speed uniformity and loss. From the analysis of the uniformity of the outlet pressure, the FLUID1 pressure uniformity at the outlet is excellent with FLUID2.However, when considering the overall compression of the die, FLUID2 can also be selected. The suitable coating style depends on the different craft and coating skill.

\section{The working principle of extrusion die}

The working principle of extrusion die is shown in Figure 1 and Figure 2.The slurry is sent steadily to the bottom or the side inlet of the cavity 1 through the feeding system. After the dispersion and distribution in the cavity 1, the coating slurry flows into cavity 2 from the slit formed by upper, lower dies and the gasket for redistribution and redispersion, which homogenizes the pressure and velocity distribution along the spray width direction.

After the second homogenization, the slurry enters the slot for the next homogenization and is finally extruded from the lip and coated on the foil ${ }^{[1]}$.A special meniscus is formed at the junction of the foil and the paint. Lithium batteries are currently coated with a dry thickness between 50 200um and a wet thickness between 100 300um. The coating thickness is related to the thickness of the gasket. A thinner coating thickness requires a smaller coating gap, which means the machine needs a thinner gasket. Fluid discharges difficultly and the foil is easily broken with a thinner gasket, which results a coating defect. In general, the thickness of the sheet is maintained between $100 \sim 300 \mu \mathrm{m}$, and the data depends on the coating process requirements. 


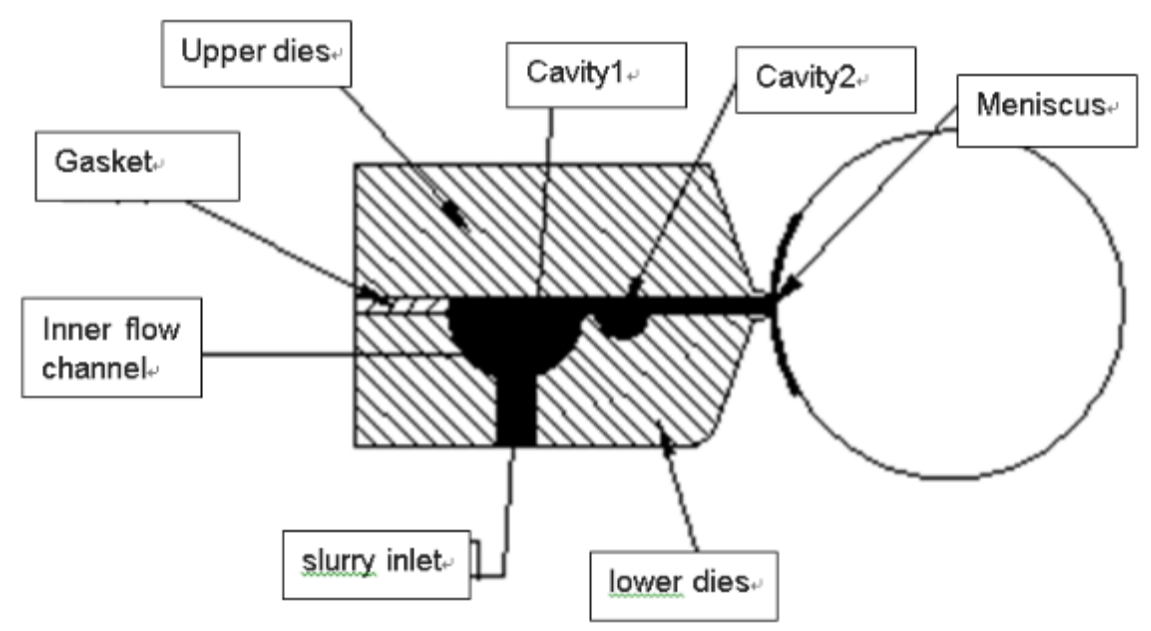

Fig.1 Work Principle of the FLUID1 Extrusion Die

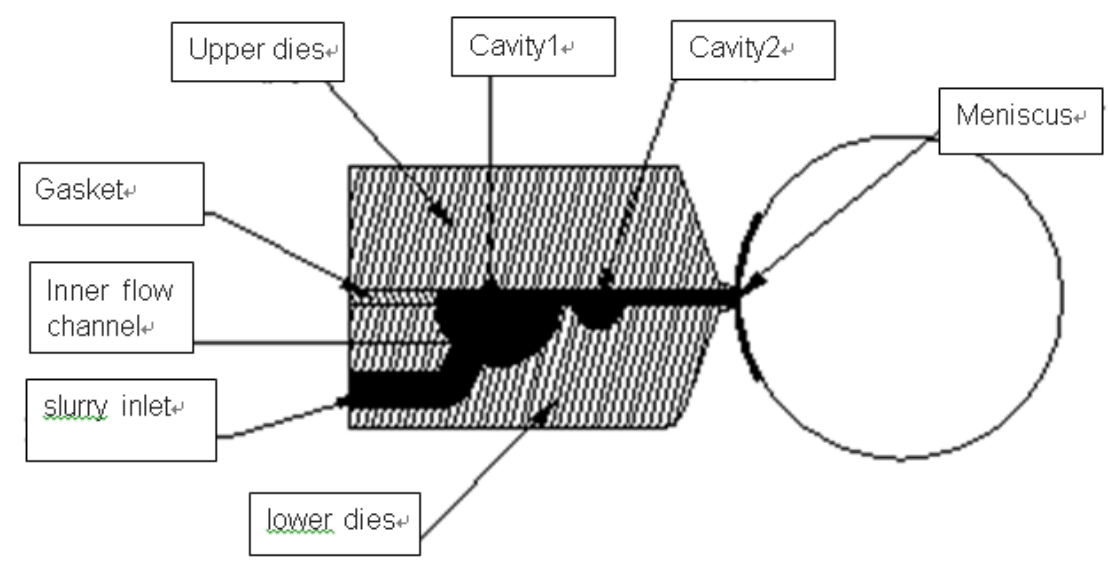

Fig.2 Work Principle of the FLUID2 Extrusion Die

\section{Boundary Conditions of the Model}

Assume that the slurry has a density of $1.45 \mathrm{Kg} / \mathrm{m} 3$, a viscosity of $1000 \mathrm{mPa} \cdot \mathrm{s}$ and a solid content of the slurry is 55\%.The coating flow path includes a single inlet and outlet, and the runner inlet is the speed import and the speed is $0.6 \mathrm{~m} / \mathrm{s}$. The outlet is a pressure outlet with standard atmospheric pressure. The wall is set to be a mark and the speed is 0 . Surface tension of the slurry is $0.0417 \mathrm{~N} / \mathrm{m}$. Because the velocity disturbance of the slurry in the flow field is not only affected by the viscous force, its temperature, pressure, vibration and other factors also affect the flow velocity. Therefore, the slurry flow state is very complicated. In this paper, the turbulence model is used to simulate the slurry solution, and the second-order upwind and SIMPLE algorithm are used for simulation calculation $^{[2-3]}$. 
The formulas for dissipation rate $\varepsilon$ and turbulent kinetic energy $\mathrm{k}$ in turbulent transport are as follows:

$$
\begin{aligned}
& \operatorname{Re}=\bar{u} D_{H} / \eta \\
& D_{H}=A / x \\
& I=0.16(\mathrm{Re})^{-1 / 8} \\
& \varepsilon=C_{u}^{3 / 4} \frac{k^{3 / 2}}{l} \\
& k=\frac{3}{2}(\bar{u} I)^{2} \\
& l=0.07 L
\end{aligned}
$$

In the formula:

$\overline{\mathrm{u}}$ : average velocity of the slurry;

Re: Reynolds number;

DH: hydraulic diameter;

A: flow area;

$\eta$ : viscosity of the fluid;

$\mathrm{X}$ : wet period;

$l$ : length of the turbulence;

I: turbulence intensity.

Empirical constant $\mathrm{Cu}=0.0845$.

$\overline{\mathrm{u}}=0.6 \mathrm{~m} / \mathrm{s}, \eta=3 \mathrm{pa} \cdot \mathrm{s}, \mathrm{DH}=0.0144$,

The simulation software, fluent, requires hydraulic diameter and turbulence intensity. According to the above formula, the turbulence intensity is 0.5 and the hydraulic diameter is 0.0144 .

\section{Simulation Results Analysis}

The uniformity and size of the export velocity and the outlet pressure directly affects the quality of the coating. Therefore, the export parameters of the two models are analyzed and compared.

\subsection{Analysis of Velocity Simulation Results}

The velocity of the die outlet is the key to study the inner flow path in the whole mold head, and the outlet velocity cloud chart and velocity streamline are shown below. 


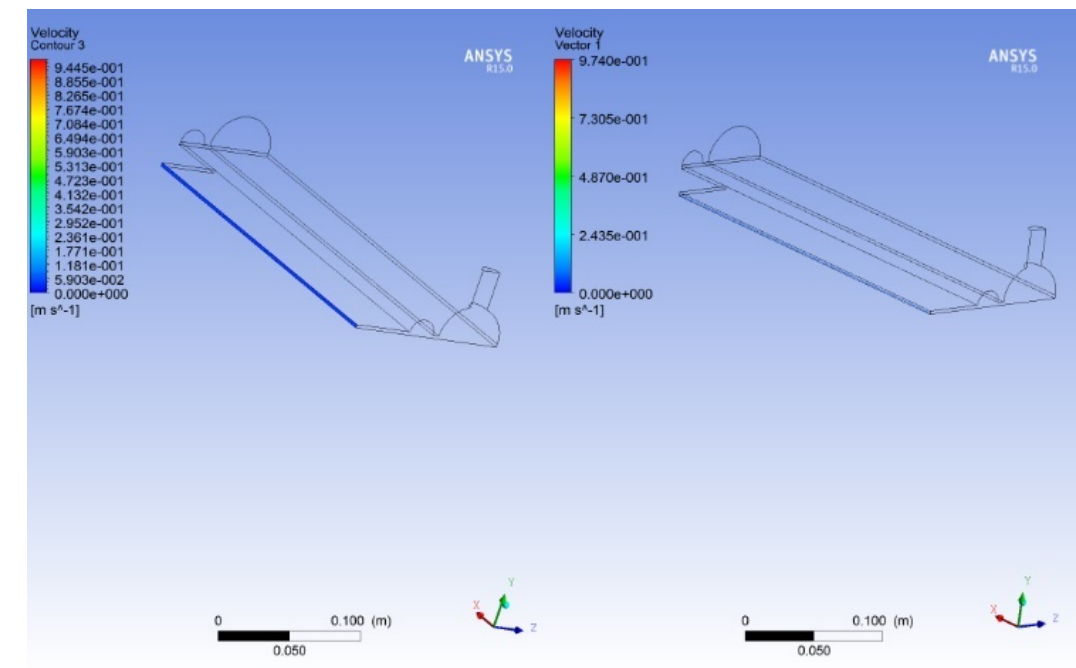

Fig.6 FLUID1 outlet velocity cloud map and velocity streamline diagram

FLUID1 outlet velocity cloud and velocity streamline is as shown in Figure 6. The velocity in the outlet direction is basically the same, and its velocity is much faster than the velocity in the width direction of the cavity 1 and the cavity 2, what's more, the slurry is evenly distributed at the outlet of the cavity. According to the simulation results, it can be known that the slots further spread and homogenize the slurry velocity along the width direction.

In FLUID1, the coating slurry outflows from the outlet, which determines the horizontal velocity of the coated beads. In the ideal state, the velocity of the width and the vertical direction is zero, and the horizontal direction is the direction of the total velocity.

From the line chart, the velocity in the $\mathrm{U}$ and $\mathrm{V}$ directions approaches 0 at the outlet of the inner flow channel, and is basically equal to the total velocity along the $\mathrm{W}$ direction. The velocity changes smoothly, which means no vortex and violent shaking phenomenon. W equals to the horizontal direction, $\mathrm{V}$ equals to the vertical direction and $\mathrm{U}$ equals to the width direction. The average value of the total velocity is $0.066 \mathrm{~m} / \mathrm{s}$ and the basic deviation is 0.0031 . The uniformity of the coating slurry is higher than 0.0066 and 0.0061 in the cavity 1 and 0.0058 and 0.0052 in the cavity 2, which also proves that the homogenization is carried again. The uniformity of the outlet coating slurry can reach the coating requirements. The average velocity in $\mathrm{W}$ direction is $0.0659 \mathrm{~m} / \mathrm{s}$, the basic deviation is 0.0030 and the average velocity in $U$ direction is $0.0002 \mathrm{~m} / \mathrm{s}$. The basic deviation is 0.0036 , the average value in $\mathrm{V}$ direction velocity is $0.00009 \mathrm{~m} / \mathrm{s}$, and the basic deviation is 0.0011 . The outlet status is basically close to the ideal state and meets the coating requirements.

Slurry 1 has an inlet velocity of $0.6 \mathrm{~m} / \mathrm{s}$ in FLUID1. According to the simulation structure, the average outlet velocity is about $0.066 \mathrm{~m} / \mathrm{s}$. During the flow of slurry, the velocity gradually decreases, and the outlet velocity is about 0.1 times of inlet. However, the relationship between import velocity and export velocity is complex and there is no direct linear relationship. Only rough estimates can be made. 


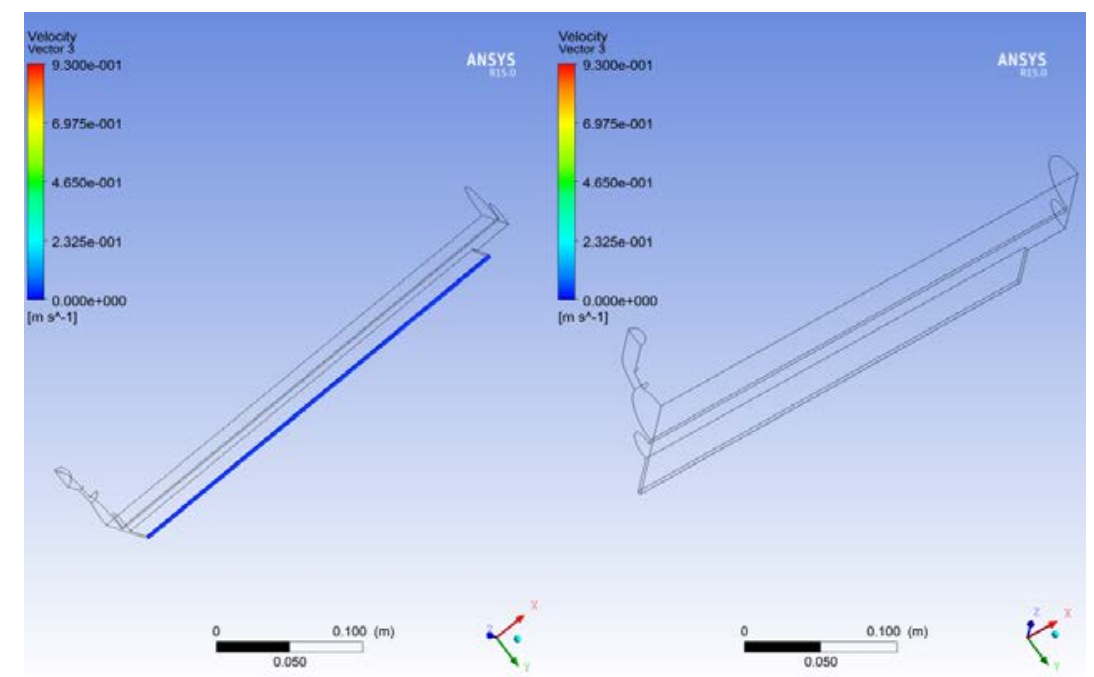

Fig. 7 FLUID2 outlet velocity cloud map and velocity streamline diagram

FLUID2 outlet velocity cloud map and velocity streamline is as shown in Figure 7. In the ideal state, the total velocity at the outlet equals to the velocity in the horizontal direction, and the velocity in the vertical and width directions is zero. The average value of the total velocity at the outlet is 0.056 $\mathrm{m} / \mathrm{s}$, the uniformity is 0.008 .The average on the U-direction and V-direction is $0.0004 \mathrm{~m} / \mathrm{s}$ and $0.0005 \mathrm{~m} / \mathrm{s}$, also, the uniformity is 0.001 and 0.002 . The average value in the $\mathrm{W}$ direction is 0.055 $\mathrm{m} / \mathrm{s}$ and the uniformity is 0.01 . From the line chart, the velocity on UW is $0.004 \mathrm{~m} / \mathrm{s}$ and $0.0005 \mathrm{~m} / \mathrm{s}$. The velocity on $\mathrm{W}$ direction is $0.055 \mathrm{~m} / \mathrm{s}$, which is basically closed to the total velocity of $0.056 \mathrm{~m} /$ $\mathrm{s}$, this result basically meets the ideal outlet status. This also proves that the homogenization is carried again, and the uniformity of the outlet coating slurry can reach the coating requirements Take 100 points randomly at the FLUID1 and FLUID2 outlet planes, output and analyze the velocity and the following broken line diagram is drawn.

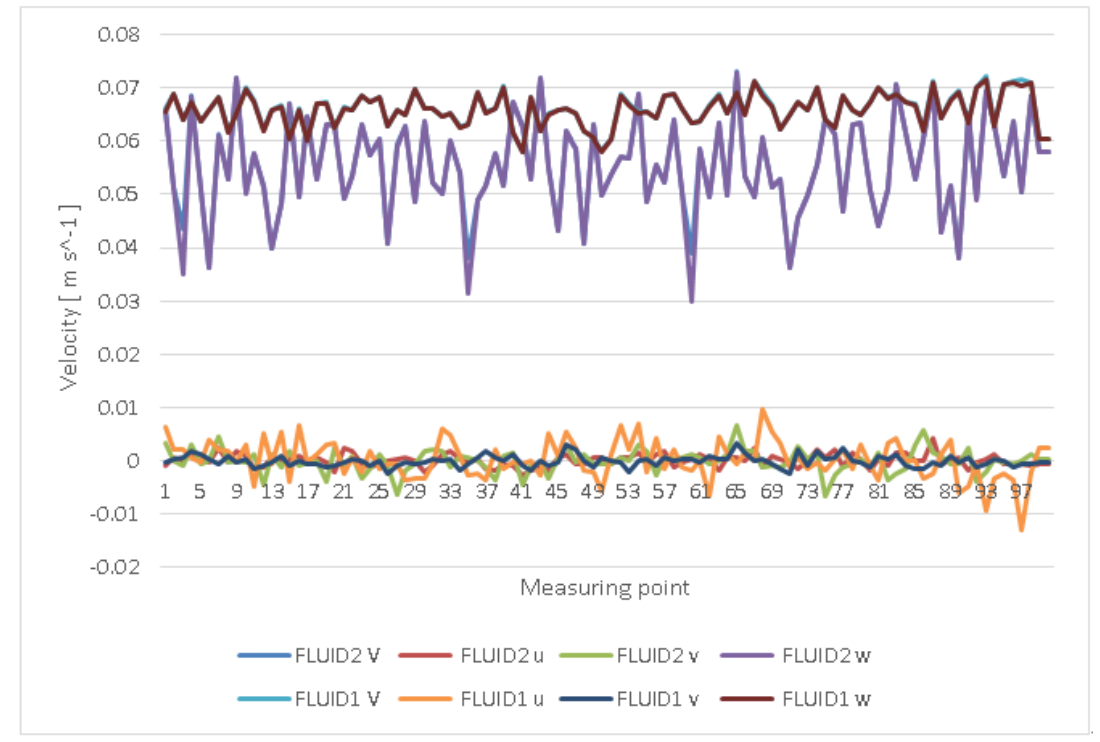

Fig. 8 FLUID1, FLUID2 velocity analysis at Out surface 
From the line chart 8 , the average velocity of the outlet of FLUID1 reaches $0.66 \mathrm{~m} / \mathrm{s}$ when the inlet velocity is $0.6 \mathrm{~m} / \mathrm{s}$, which is slightly higher than $0.56 \mathrm{~m} / \mathrm{s}$ of FLUID2. The uniformity of FLUID1 reaches 0.003 , which is much better than the FLUID2 uniformity of 0.008 . It can be seen that the FLUID1 has better uniformity of the total velocity than the FLUID2. FLUID1 outlet velocity basically coincides with the total velocity in the $\mathrm{W}$ direction, and FLUID2 has bumps at three different positions, which affects the overall stability of the velocity. The UV direction velocity of both coating methods is 0 , and the uniformity in all directions can be basically satisfied. For coating requirements, the FLUID1 die is superior to the FLUID2 die in terms of velocity uniformity and loss.

\subsection{Results Analysis of the Pressure Simulation}

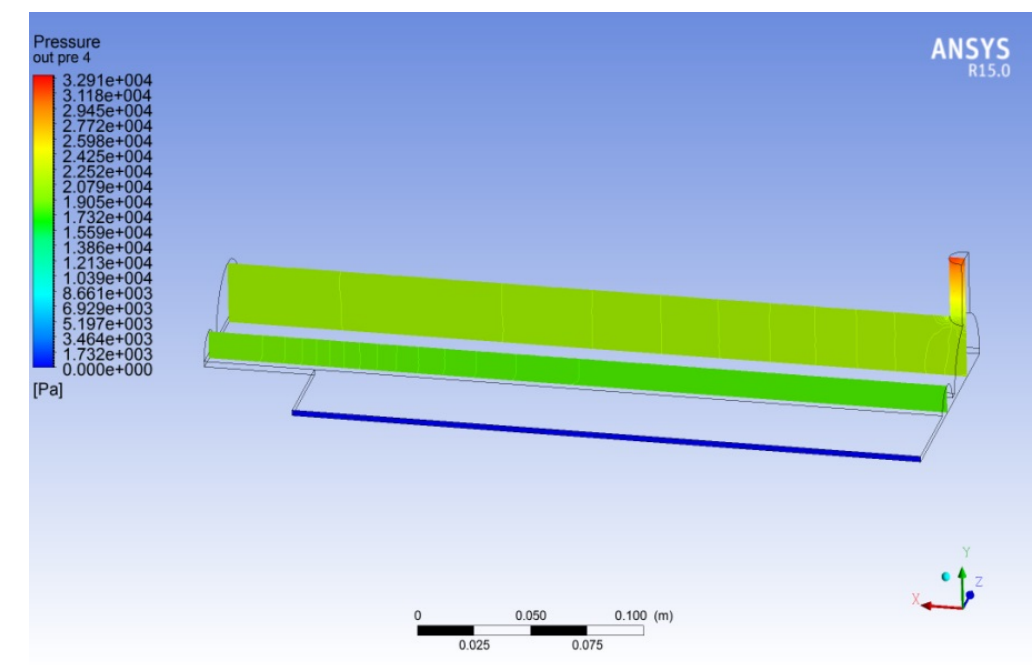

Fig9. Pressure nephogram of the Plane 1and 2 in FLUID1

As shown in Figure 9, the pressure distribution is very even with respect to the velocity. a quantitative measure of pressure at three planes, Its distribution is very even distribution. Take 100 measure points randomly at Plane1, Plane2 and out planesas, then output the pressure value, and draw it as a line graph as shown above.

The maximum pressure is closed to $28897 \mathrm{~Pa}$ in the plane of the cavity 1 , its average pressure value is $21177 \mathrm{~Pa}$, and the pressure uniformity is 889 . In addition, it is also known through calculations, the pressure nephogram is distributed more sparsely in the corner of the cavity 1 . After the diffusion and homogenization, the pressure distribution has been uniformed in the cavity2.The maximum pressure value is $19039 \mathrm{~Pa}$, the average pressure is $18305 \mathrm{~Pa}$, and the uniformity reaches 310 . The initial pressure of the slurry at the outlet position is 0 , the maximum pressure at the outlet is $21 \mathrm{~Pa}$ after 100 iterations calculation, and the average pressure is about 5Pa.Compared with the uniformity in cavity 1 and cavity 2, pressure at the outlet is more uniform. The negative pressure at the outlet can help to form the coating step beads. Some manufacturers will increase the negative pressure device in the coating to achieve the coating effection. 


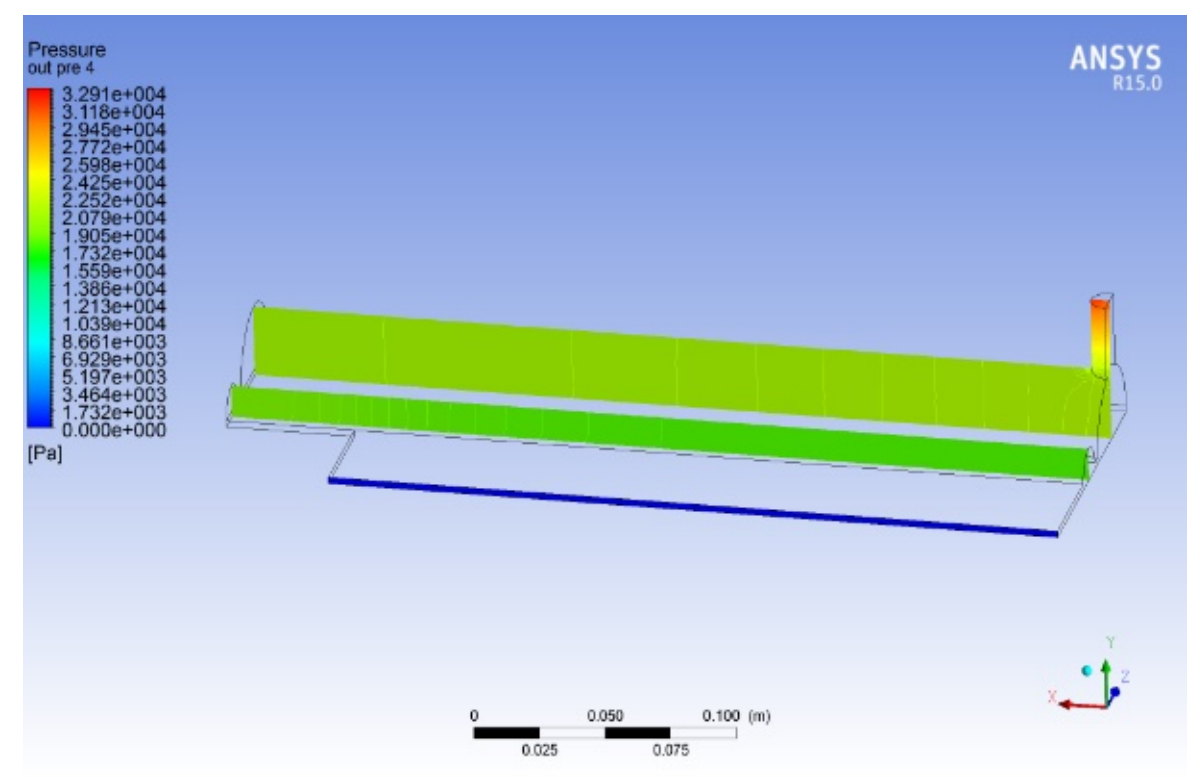

Fig10. Pressure nephogram of the Plane 1and 2 in FLUID2

As shown in Figure 10, its inlet pressure is significantly higher than the other position, and the maximum value is $32910 \mathrm{~Pa}$. When getting into the cavity 1 , the pressure gradually decreased to about 20790Pa.The uniform pressure is much uniform because of the pressure in Cavity 2, which can meet the coating requirements. To describe the pressure distribution intuitively, 100 measurement points are taken as the experiment value. Draw it as a line graph as shown in Figure 11.

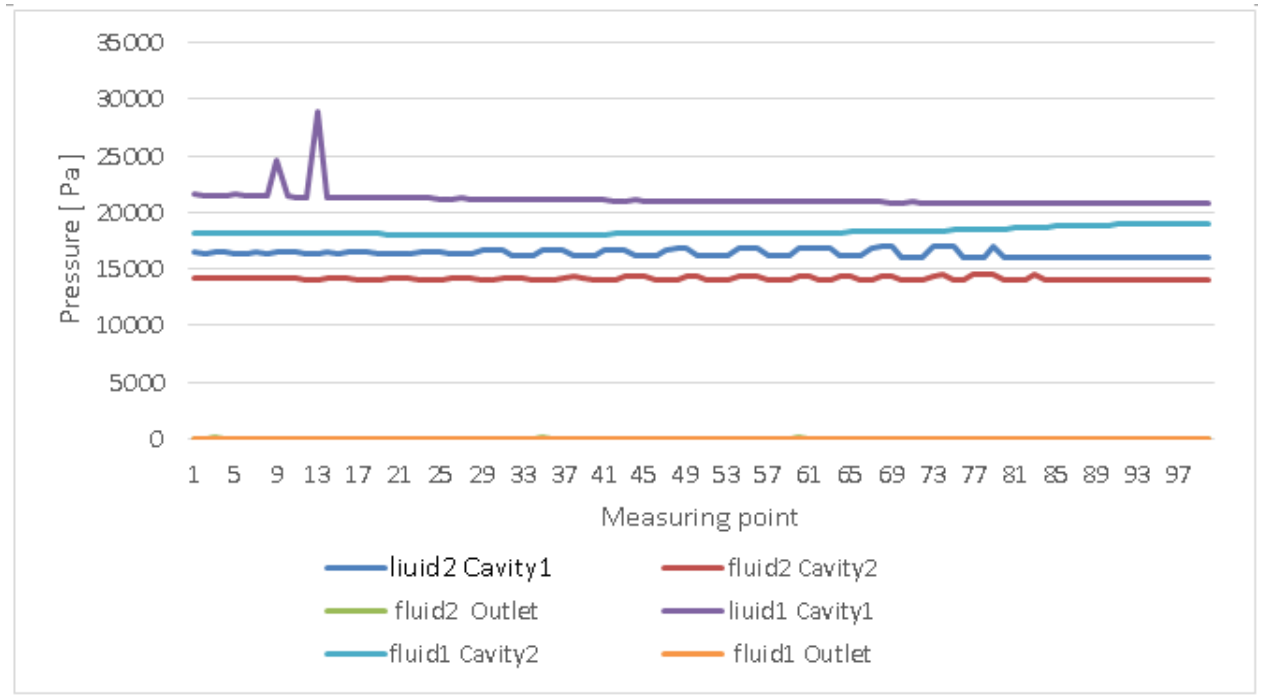

Fig.11 Measure Points Pressure on Plane1, Plane2 and Out

From the line chart 11, the average pressure of FLUID1 achieves 21173Pa in the cavity 1, which is higher than the FLUID2 average pressure of 16380Pa.The uniformity is 885 in FLUID1, which is higher than the uniformity 307 in FLUID2. It can be seen that the pressure uniformity in FLUID1 is slightly worse than FLUID2. The average pressure of FLUID1 in cavity 2 reaches 18,312 Pa, higher than the average pressure 14155Pa of FLUID2.Besides, the uniformity of FLUID1 reaches 317, 
higher than the FLUID2. As a result, the pressure and uniformity of FLUID1 in cavity 2 is still poor than FLUID2. The average pressure at the FLUID1 outlet is about 5Pa, lower than the average pressure of the FLUID2. Also, the FLUID1's uniformity value is lower than FLUID2.

Take the uniformity analysis as the outlet pressure, FLUID1 is obviously better than FLUID2. However, when it comes to the overall compression of the die, the FLUID2 is also a suitable plan. The suitable coating style depends on the different craft and coating skill. This paper only analyzes the speed and pressure of two coating equipments in the coating process, and this way applies to the selection of coating equipment from the perspective of speed and pressure. In the actual coating process, the coating quality is determined by various factors such as the viscosity of the slurry, the humidity, the solid content, the coating speed, the thickness of the gasket, etc. Different slurry matches different equipment ${ }^{[4]}$.

\section{Conclusion}

In the case of import speeds of $0.6 \mathrm{~m} / \mathrm{s}$, the average exit speed of FLUID1 reaches $0.66 \mathrm{~m} / \mathrm{s}$, higher than FLUID2 $0.56 \mathrm{~m} / \mathrm{s}$. The uniformity of FLUID1 reaches 0.003 , far better than the FLUID2 uniformity of 0.008 . Therefore, the overall velocity uniformity of FLUID1 is better than that of FLUID2. The velocity in the W direction of the FLUID1's exit velocity basically coincides with the total velocity. FLUID2 has bumps at three different locations that affects the overall stability of speed. The direction speed of both coating methods UV is basically 0 , and the uniformity in all directions can basically meet the coating requirements, the FLUID1 die is superior to the FLUID2 die by considering the uniformity and loss of speed.

The average pressure of the FLUID1 outlet is about 5Pa lower than the FLUID2 25Pa, FLUID1's uniformity reaches 21 Pa below the FLUID2 of 120, only from the analysis of the uniformity of outlet pressure, the pressure uniformity of the FLUID1 outlet is excellent with FLUID2. Selection of coating quality of FLUID1 die higher than FLUID2, but when considering the overall compression of the die, FLUID2 can also be selected. The pressure of FLUID2's cavity 1 and cavity 2 are smaller than FLUID1. Which kind of coating method is more suitable for positive and negative film slurry coating, which should be selected according to different processes and coating technology requirements.

\section{References}

[1] ZHOU Yunfu. Research on Lithium Power Battery Pole Piece Extrusion Coating Head [D].Southeast University, 2014.5.

[2] HE Wei. Flow Simulation of Coating Machine [D]. Hefei University of Technology, 2011.4.

[3] BAO Nengsheng, LIU Xiaoshan, MA Wan, etc. Numerical Calculation and Analysis on Roll Coating Process of the Clearance between Two Rollers [J]. Packaging Engineering, 2016(23):6-12.

[4] Kang H, Park J, Shin K. Statistical analysis for the manufacturing of multi-strip patterns by roll-to-roll single slot-die systems [J]. Robotics and Computer Integrated Manufacturing, 2014, 30(4):363-368. 